\title{
Metformin attenuates LPS-induced neuronal injury and cognitive impairments by blocking NF-kB pathway
}

Chenliang Zhou ${ }^{1 \dagger}$, Bo Peng ${ }^{2 \dagger}$, Zhenghui Qin ${ }^{3}$, Wei Zhu ${ }^{3^{*}}$ and Cuiping Guo ${ }^{\text {* }^{*}}$

\begin{abstract}
Background: Neuroinflammatory response is considered to be a high-risk factor for cognitive impairments in the brain. Lipopolysaccharides (LPS) is an endotoxin that induces acute inflammatory responses in injected bodies. However, the molecular mechanisms underlying LPS-associated cognitive impairments still remain unclear.

Methods: Here, primary hippocampal neurons were treated with LPS, and western blotting and immunofluorescence were used to investigate whether LPS induces neurons damage. At the same time, SD rats were injected with LPS $(830 \mu \mathrm{g} / \mathrm{Kg}$ ) intraperitoneally, and Open field test, Novel Objective Recognition test, Fear condition test were used to detect cognitive function. LTP was used to assess synaptic plasticity, and molecular biology technology was used to assess the NF-kB pathway, while ELISA was used to detect inflammatory factors. In addition, metformin was used to treat primary hippocampal neurons, and intraventricularly administered to SD rats. The same molecular technics, behavioral and electrophysiological tests were used to examine whether metformin could alleviate the LPS-associated neuronal damage, as well as synaptic plasticity, and behavioral alterations in SD rats.
\end{abstract}

Results: Altogether, neuronal damage were observed in primary hippocampal neurons after LPS intervention, which were alleviated by metformin treatment. At the same time, LPS injection in rat triggers cognitive impairment through activation of NF-KB signaling pathway, and metformin administration alleviates the LPS-induced memory dysfunction and improves synaptic plasticity.

Conclusion: These findings highlight a novel pathogenic mechanism of LPS-related cognitive impairments through activation of NF-kB signaling pathway, and accumulation of inflammatory mediators, which induces neuronal pathologic changes and cognitive impairments. However, metformin attenuates LPS-induced neuronal injury and cognitive impairments by blocking NF-KB pathway.

Keywords: Lipopolysaccharides (LPS), Metformin, NF-kB, Neuronal injury, Cognitive impairments

*Correspondence: qixi202081@163.com; rm003778@whu.edu.cn ${ }^{\dagger}$ Chenliang Zhou and Bo Peng contributed equally to this work

${ }^{1}$ Department of Critical Care Medicine, Renmin Hospital of Wuhan University, Wuhan, China

${ }^{3}$ Department of Critical Care Medicine, Tianyou Hospital Affiliated to Wuhan University of Science and Technology, Wuhan, China Full list of author information is available at the end of the article

\begin{abstract}
Introduction
Neuroinflammation is a double-edged sword. On one hand, inflammatory mechanisms are beneficial in the repair of nervous system injury in certain circumstances. However, on the other hand, prolonged neuroinflammation could induce or at least exacerbate neurodegenerative diseases [1-3]. Clinical studies and animal experiments have shown that neuroinflammation in the brain is closely related to the occurrence and
\end{abstract}

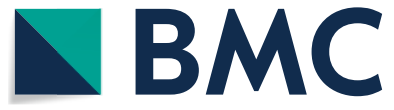

(c) The Author(s) 2021. Open Access This article is licensed under a Creative Commons Attribution 4.0 International License, which permits use, sharing, adaptation, distribution and reproduction in any medium or format, as long as you give appropriate credit to the original author(s) and the source, provide a link to the Creative Commons licence, and indicate if changes were made. The images or other third party material in this article are included in the article's Creative Commons licence, unless indicated otherwise in a credit line to the material. If material is not included in the article's Creative Commons licence and your intended use is not permitted by statutory regulation or exceeds the permitted use, you will need to obtain permission directly from the copyright holder. To view a copy of this licence, visit http://creativecommons.org/licenses/by/4.0/. The Creative Commons Public Domain Dedication waiver (http://creativeco mmons.org/publicdomain/zero/1.0/) applies to the data made available in this article, unless otherwise stated in a credit line to the data. 
development of various acute and chronic neurodegenerative diseases such as Parkinson's disease (PD) $[4,5]$, Alzheimer's disease (AD) [6,7] and multiple sclerosis (MS) $[8,9]$. However, the role of neuroinflammation in the progression of neurodegenerative diseases is very complex and many problems are far from being clear.

Metformin, a biguanide class of antidiabetics, has shown promise in the management of cognitive impairments [10, 11], as its administration was found to improve mild cognitive impairments in a clinical study [12]. It is well known that cognitive function could be impaired by variousassaults, including inflammation. Neuroinflammation plays an important role in the pathogenesis of cognitive dysfunction after lipopolysaccharides (LPS) exposure [13, 14]. Metformin was reported to improveschizophrenia-like cognitive deficits induced by LPS exposure and reduces LPS-induced fear memory reconsolidation impairments and ameliorated inflammation [15].

$\mathrm{NF}-\mathrm{KB}$ is an important transcription factor that regulates chronic disease by promoting inflammation $[16,17]$. Activated NF-kB was detected in the substantia nigra of PD patients and PD animal models. More importantly, the substantia nigra of PD patients at autopsy showed significant co-localization of NF-kB with p65 and CD11b (markers of microglia cells), suggesting that the inflammatory response regulated by NF- $\mathrm{kB}$ plays an important role in the pathogenesis of PD $[18,19]$. Moreover, inhibition of NF- $\mathrm{KB}$ activation prevented the activation of microglia and the loss of dopaminergic neurons in PD animals [20]. Therefore, these together indicate that $\mathrm{NF}-\kappa \mathrm{B}$ may be an important therapeutic target in the treatment of neuroinflammatory and neurodegenerative diseases. We therefore, hypothesized that metformin might alleviate LPS-induced cognitive impairments, and NF-кB might play a key role.

In the current study, behavioral tests showed that blocking or decreasing NF- $\mathrm{kB}$ activity attenuates LPSinduced learning and memory dysfunction in rats. Moreover, metformin decreases inflammatory response in the hippocampus, restores the reduced expression of synaptic proteins and improves synaptic damage. These data suggest that treatment with metformin may contribute to the recovery of learning and memory function after LPS-induced neuronal injury and cognitive impairments, and provide an insight into clinical intervention in neuropathological and behavioral complications associated with neuroinflammatory disorders.

\section{Material and methods Reagents}

LPS was purchased from Sigma Aldrich (Escherichia coli serotype 0127:B8, Sigma Aldrich L3129). Nuclear and cytoplasmic protein preparation kit (P1200) was purchased from Pulilai, and all cell culture reagents were from Thermo Fisher Scientific. Rat IL-1 $\beta$ (Interleukin 1 Beta) ELISA Kit (E-EL-R0012c), Rat IL-6(Interleukin 6) ELISA Kit (E-EL-M0044c), Rat TNF- $\alpha$ (Tumor Necrosis Factor Alpha) ELISA Kit (E-EL-R2856c) were purchased from Elabscience Biotechnology.

\section{Animals}

Male Sprague-Dawley (SD) rats (2 months old) were supplied by Liaoning Changsheng Biotech Co. Ltd. Rats were fed with normal rats' food and kept under 12-h light and 12-h dark cycle with free water access. Rats were randomly assigned to different experimental groups and treated according to the experimental requirements. LPS $(830 \mu \mathrm{g} / \mathrm{Kg})$ was injected intraperitoneally (i.p.) and an equal volume of vehicle (normal saline) was used as a control. Behavioral, electrophysiological, and biochemical tests were performed at $6 \mathrm{~h}$ after injection (Fig. 1A). Metformin has might have a certain therapeutic potential for cognitive impairments. To further investigate on this, Healthy SD rats were randomly divided into three groups, the control group (control group), model group (LPS group) and intervention group (Met + LPS group). The Met+LPS group were injected with metformin $10 \mathrm{mM}$ into lateral ventricle and LPS $(830 \mu \mathrm{g} / \mathrm{Kg})$ injection intraperitoneally. Behavioral, electrophysiological, and biochemical tests were performed at $6 \mathrm{~h}$ after injection (Fig. 5A).

\section{LPS model}

LPS $(830 \mu \mathrm{g} / \mathrm{Kg})$ was injected intraperitoneally (i.p.) and an equal volume of vehicle (normal saline) was used as a control. Behavioral and electrophysiological tests were performed at $6 \mathrm{~h}$ after injection. After completion of the tests, the animals were sacrificed, the hippocampi were quickly removed, immediately frozen on dry ice and stored at $-80^{\circ} \mathrm{C}$ until further molecular analysis [21].

\section{Lateral ventricle stereotactic surgery}

Rats were anesthetized with isoflurane and placed on a stereotactic device. Bilateral holes were drilled in the skull stereotaxically at the following coordinates: posterior $1.2 \mathrm{~mm}$, lateral $2.6 \mathrm{~mm}$, and depth $4.0 \mathrm{~mm}$ relative to bregma. Concentration of $5 \mathrm{mM}$ and $10 \mathrm{mM}$ metformin $(5 \mu \mathrm{L})$ were injected into the lateral ventricles at a rate of $0.25 \mu \mathrm{L} / \mathrm{min}$. Vital signs of the rats were monitored. After the injection, the needle was allowed to stay for two minutes and then slowly pulled out. 


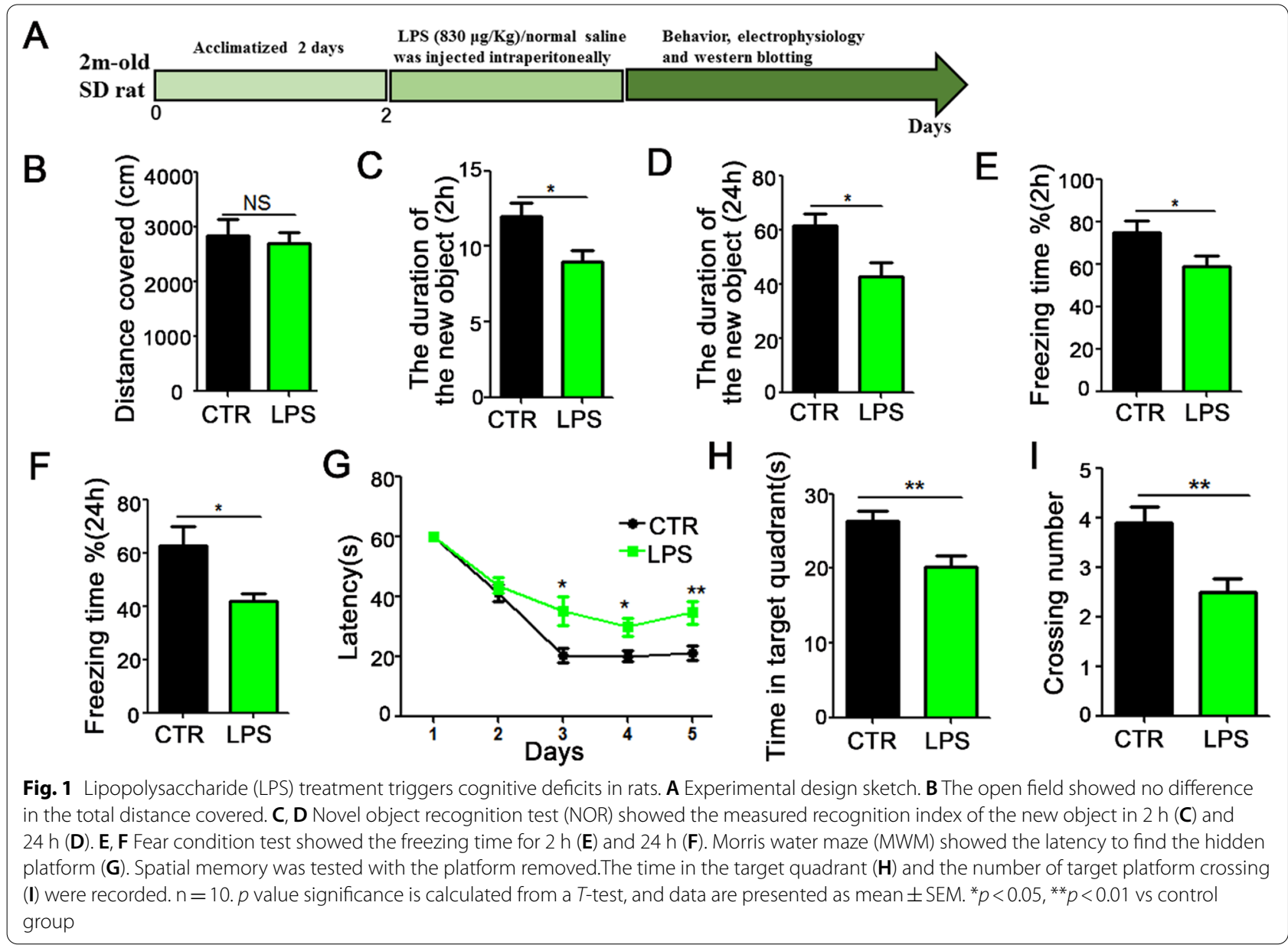

\section{Behavior tests \\ Open field test}

The rats were put into the laboratory and stayed for two days, and the rats were placed alone in the test box $(100 \mathrm{~cm} \times 100 \mathrm{~cm} \times 70 \mathrm{~cm}$ container $)$ for spontaneous activity for $5 \mathrm{~min}$. The distance traveled was tracked and measured to evaluate their locomotor ability.

\section{Novel objective recognition test}

In the same way as open field test, the rats were placed in the laboratory and allowed to stay for one day, before the experimental test was carried out. Object A and $B$ were placed on the two corners of the box, rats are placed in center of the box and allowed to freely explore for $5 \mathrm{~min}$. After $2 \mathrm{~h}$, object B was replaced by the new object $C$, the rats are placed in the box for another $5 \mathrm{~min}$. After $24 \mathrm{~h}$, object $\mathrm{C}$ was replaced by object $\mathrm{D}$, and the rats were again placed in the box for 5 min. The exploration time of objects A, B, C and D were recorded during the activity.

\section{Fear conditioning test}

Similarly, the rats were put into the testing room one day earlier for adaptation. The experiment was divided into two stages. In the first stage (training), rats were placed in a test box for $3 \mathrm{~min}$, followed by $10 \mathrm{~s}$ of sound stimulation, and immediately followed by electrical shock stimulation $(0.8 \mathrm{~mA}, 3 \mathrm{~s})$, repeatedly three times. In the second stage (test), following stimulation completion, the test is performed at $2 \mathrm{~h}$ and $24 \mathrm{~h}$. During this process, only the sound stimulus was given, without electrical shock stimulation. The freezing time of rats was recorded during the tests at $2 \mathrm{~h}$ and $24 \mathrm{~h} \mathrm{[22].}$

\section{Morris water maze test (MWM)}

Spatial learning and memory were tested by MWM test. The rats were put in the room a day earlier. When testing spatial learning ability, rats were placed in a water maze to find a hidden platform in $60 \mathrm{~s}$ for five consecutive days. If the rat did not find the platform within $60 \mathrm{~s}$, they were guided to the platform and allowed to stay there for $20 \mathrm{~s}$. On the 6th day, the platform was removed and spatial 
memory was tested, the number of crossing the platform position and the time spent in the target quadrant were recorded.

\section{Long-term potentiation (LTP)}

LTP was used to detect synaptic plasticity in the hippocampus, and the MED64 multi-electrode array system was used (Alpha Med Sciences, Kadoma, Japan) to detect the LTP. Before detection, the rats were quickly sacrificed and brains were quickly removed and cut. The slices were incubated in artificial cerebrospinal fluid for $30 \mathrm{~min}$, followed by three sets of high-frequency stimulation (HFS of $100 \mathrm{~Hz}$, lasting for $1 \mathrm{~s}$ ). The field excitatory postsynaptic potentials (FEPSPs) of CA1 neurons were recorded $[22,23]$.

\section{Primary cultures of hippocampal neurons}

Primary cultures of hippocampal neurons were performed as previously described [24]. Briefly, hippocampal tissue was quickly removed and placed in HANK's buffered saline and gently chopped, then suspended in a $0.25 \%(\mathrm{v} / \mathrm{v})$ trypsin solution at $37{ }^{\circ} \mathrm{C}$ for $15 \mathrm{~min}$. Neurons were plated in 6-well and 12-well plates coated with $100 \mu \mathrm{g} / \mathrm{mL}$ poly-D-lysine and supplemented with $2 \%$ $(\mathrm{v} / \mathrm{v})$ B-27 and $1 \times$ GlutaMAX. Hippocampal neurons were cultured and treated at $37{ }^{\circ} \mathrm{C}$ in a humidified $5 \%$ (vol/vol) $\mathrm{CO}_{2}$ incubator [22].

\section{Immunofluorescence}

Hippocampal neurons were fixed with $4 \%$ paraformaldehyde for $8 \mathrm{~min}$, washed three times with PBS, blocked with $3 \% \mathrm{BSA}$ and $0.5 \%$ Triton $\mathrm{X}-100$ for $30 \mathrm{~min}$, and labeled overnight with primary antibody at $4{ }^{\circ} \mathrm{C}$. The second day, the cells were rinsed in PBS for three times. The second antibody was then incubated at room temperature for $1 \mathrm{~h}$, and then rinsed in PBS for three times. The nuclei were stained with Hocest (1:1000) for $10 \mathrm{~min}$. Then the neurons were washed three times with PBS and mounted on slides. Confocal microscopy was used to detect cell morphology (LSM710, Zeiss, Germany) [22].

\section{Western blotting}

Western blotting was used to detect protein expression levels. Cell or tissue samples were lysed with RIPA containing the protease inhibitor PMSF and cocktail, and then centrifuged at $12,000 \mathrm{~g}$ for $10 \mathrm{~min}$. Supernatants were boiled in SDS loading buffer and protein separated using SDS-PAGE. Proteins were then transferred to nitrocellulose membranes. Five percent skim milk powder was used to block, and proteins of interest were labeled overnight with primary antibody at $4{ }^{\circ} \mathrm{C}$. After three times wash with washing buffer, the second antibody was incubated at room temperature for $1 \mathrm{~h}$ followed by another three washes, protein expression levels were visualized by using the Odyssey system. The primary antibodies used for Western blotting include PSD95 (1:1000; Cell Signaling Technology, USA), NR1 (1:1000; Millipore, USA), NR2B (1:1000; Millipore, USA), NR2A (1:1000; abcam, UK), GluA1 (1:1000; Millipore, USA), GluA2 (1:1000; Millipore, USA), Synapsin 1(1:1000; Millipore, USA), Synaptophysin (1:1000, sigma), actin (1:1000; Abcam), LaminB1(1:1000; Abcam), NF-кB p65(1:500; Cell Signaling Technology, USA).

The nuclear and cytoplasmic proteins preparation kit (P1200, Pulilai) was used to separate the nuclear and cytoplasmic components according to the manufacturer's procedures for subsequent experiments.

\section{Statistical analysis}

Data were expressed as mean \pm SEM and analyzed using GraphPad statistical software. The one-way ANOVA was used to determine the differences among groups. For the comparison between two groups, the Student's $t$ test was used. The significance was assessed at $p<0.05$.

\section{Results \\ Lipopolysaccharide (LPS) injection triggers cognitive deficits in rats}

LPS is a unique component in the cell wall of gram-negative bacteria [25-27]. LPS can induce a cascade reaction of immune stimulus and toxic pathophysiological activity of the body, releasing endotoxin and causing inflammation [28, 29]. Also, adult male rats injected with LPS showed inflammation in the central nervous system which might affect it normal function. To explore the effect of LPS on cognitive function, 20 healthy SD rats were randomly divided into LPS model group and control group which were respectively intraperitoneally injected with LPS $(830 \mu \mathrm{g} / \mathrm{Kg} / \mathrm{i} . \mathrm{p}, \mathrm{n}=10)$ or normal saline $(1 \mathrm{~mL} / \mathrm{Kg} / \mathrm{ip}, \mathrm{n}=10)$ for $6 \mathrm{~h}$ [21], Behavioral, electrophysiological, and biochemical tests were performed at $6 \mathrm{~h}$ after injection (Fig. 1A). The results from open field experiment showed that there was no significant difference in the total distance between the LPS group and control group (Fig. 1B). The Novel Object Recognition (NOR) test showed that the exploration duration of new object after LPS treatment was significantly reduced at $2 \mathrm{~h}$ (Fig. 1C) and $24 \mathrm{~h}$ (Fig. 1D). Fear condition results showed that the freezing duration after LPS treatment was significantly lower than the control group at $2 \mathrm{~h}$ (Fig. 1E) and $24 \mathrm{~h}$ (Fig. 1F). Finally, Morris water maze (MWM) was used to test memory and learning abilities, and the result showed that the LPS rats have significantly increased latency to find the hidden platform (Fig. 1G). On the day 6 , the platform was removed to test the spatial memory, both the time in the target quadrant (Fig. 1H) 
and the number of target platform crossing (Fig. 1I) were decreased. Taken together, these results suggest that LPS causes learning and memory impairments.

\section{LPS treatment induces synaptic dysfunction in rats}

A large number of studies have shown that the hippocampus (HIP) plays a very important role in learning and memory function [30-32]. To investigate how LPS induces learning and memory deficits, we explored whether hippocampal-dependent synaptic plasticity was impaired by recording long-term potentiation (LTP). The LTP test showed that the field excitatory postsynaptic potential (fEPSP) slope of the LPS group was decreased after high frequency stimulation (HFS)
(Fig. 2A and B). In addition, we examined molecular changes in synapse-related proteins in the hippocampus. Western blotting (Fig. 2C) results showed that the down-regulation of presynaptic proteins synapsin (SYN) and synaptophysin (SYT) and postsynaptic protein NR2B in the LPS group (Fig. 2D). This is an indication that LPS leads to cognitive dysfunction through the alteration of synaptic function seen as decrease of both synaptic proteins and fEPSP.

\section{LPS treatment results in synaptic dysfunction in rat primary hippocampal neurons}

LPS treatment induced cognitive impairments in rats, but whether or not it resulted in neuronal damage
A

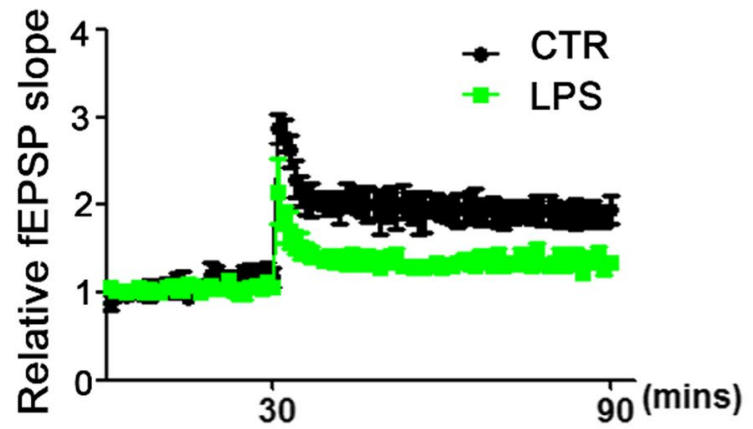

B

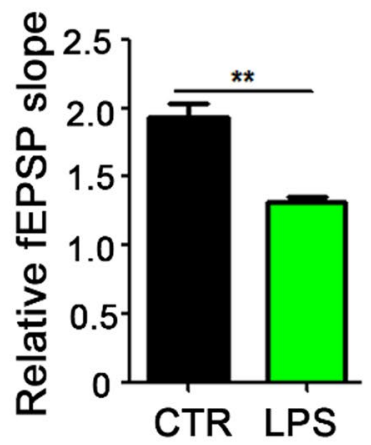

C

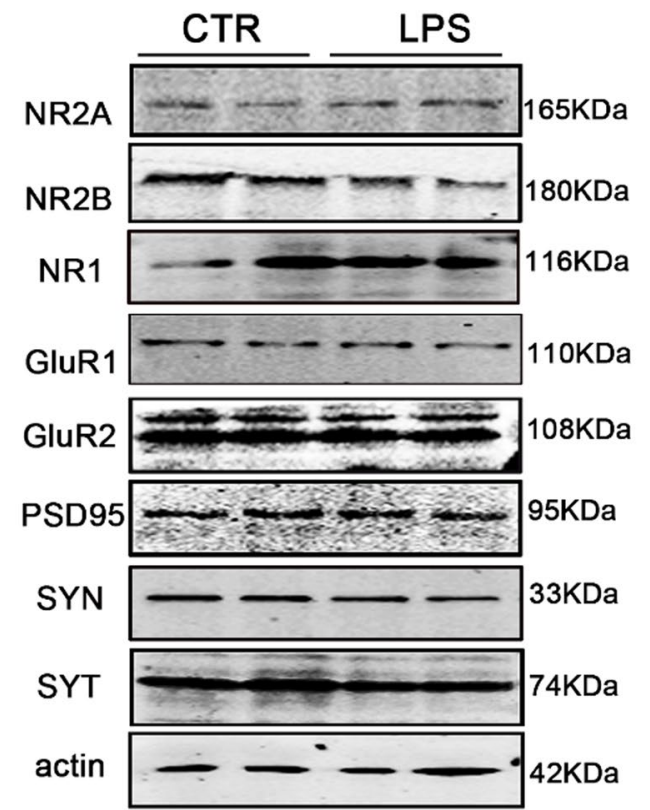

D

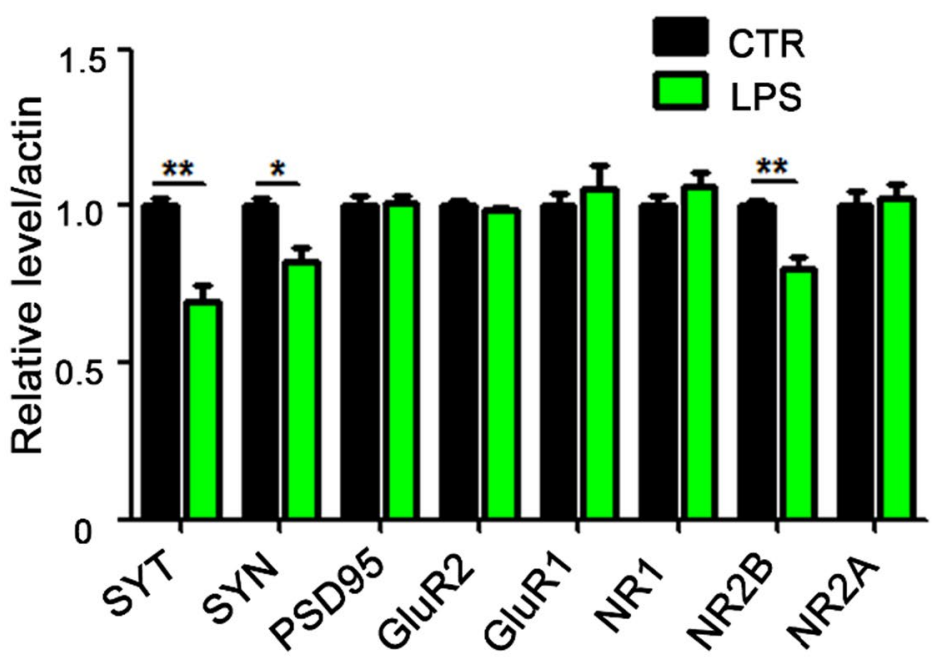

Fig. 2 LPS treatment induces synaptic dysfunction in rats. A, B Hippocampal CA3-CA1 LTP and its quantification (A) were recorded using the MED64 system. Normalized CA3-CA1 fEPSP mean slope recorded from the CA1 dendritic region in hippocampal slices (B). C, D Brain tissues from hippocampi were homogenized, and synaptic protein levels were detected by immunoblotting. $n=3$. $p$ value significance is calculated from a T-test, and data are presented as mean \pm SEM. ${ }^{*} p<0.05,{ }^{* *} p<0.01$ vs control group 
has not been clarified. To investigate the underlying mechanism based on synaptic morphology, primary hippocampal neurons were treated with LPS $(1 \mu \mathrm{g} /$ $\mathrm{ml}$ ) for $6 \mathrm{~h} \mathrm{[33].} \mathrm{Western} \mathrm{blotting} \mathrm{showed} \mathrm{a} \mathrm{downregu-}$ lation in the levels of pre-synaptic proteins SYT and postsynaptic protein PSD95 (Fig. 3A and B). In addition, anti-MAP2 antibody was used to evaluate neuronal morphology (Fig. 3C). When compared with the control group, the complexity of dendritic branches was reduced after LPS treatment at all points above $40 \mu \mathrm{m}$ away from the cell body (Fig. 3D), as well as the total length of dendrites (Fig. 3E). These results suggest that LPS-induced cognitive impairments could be the reflection of synapticalterations.
Metformin pretreatment attenuates LPS-induced synaptic dysfunction in rat primary hippocampal neurons

Metformin has shown promise for cognitive impairments in the treatment of diabetes. We therefore, hypothesized that metformin might alleviate LPS-inducedsynaptic dysfunction. To investigate the role of metformin in rat primary hippocampal neurons with LPS treatment, metformin $5 \mathrm{mM}$ was used to pretreat the primary hippocampal neurons prior to their stimulation with LPS $(1 \mu \mathrm{g} / \mathrm{ml})$ for $6 \mathrm{~h}[33,34]$. Western blotting results showed that metformin attenuated LPS-induced downregulation of PSD95 (Fig. 4A and B). Immunofluorescence results (Fig. 4C) showed the dendritic complexity at all points farther than $60 \mu \mathrm{m}$ from the cell body was
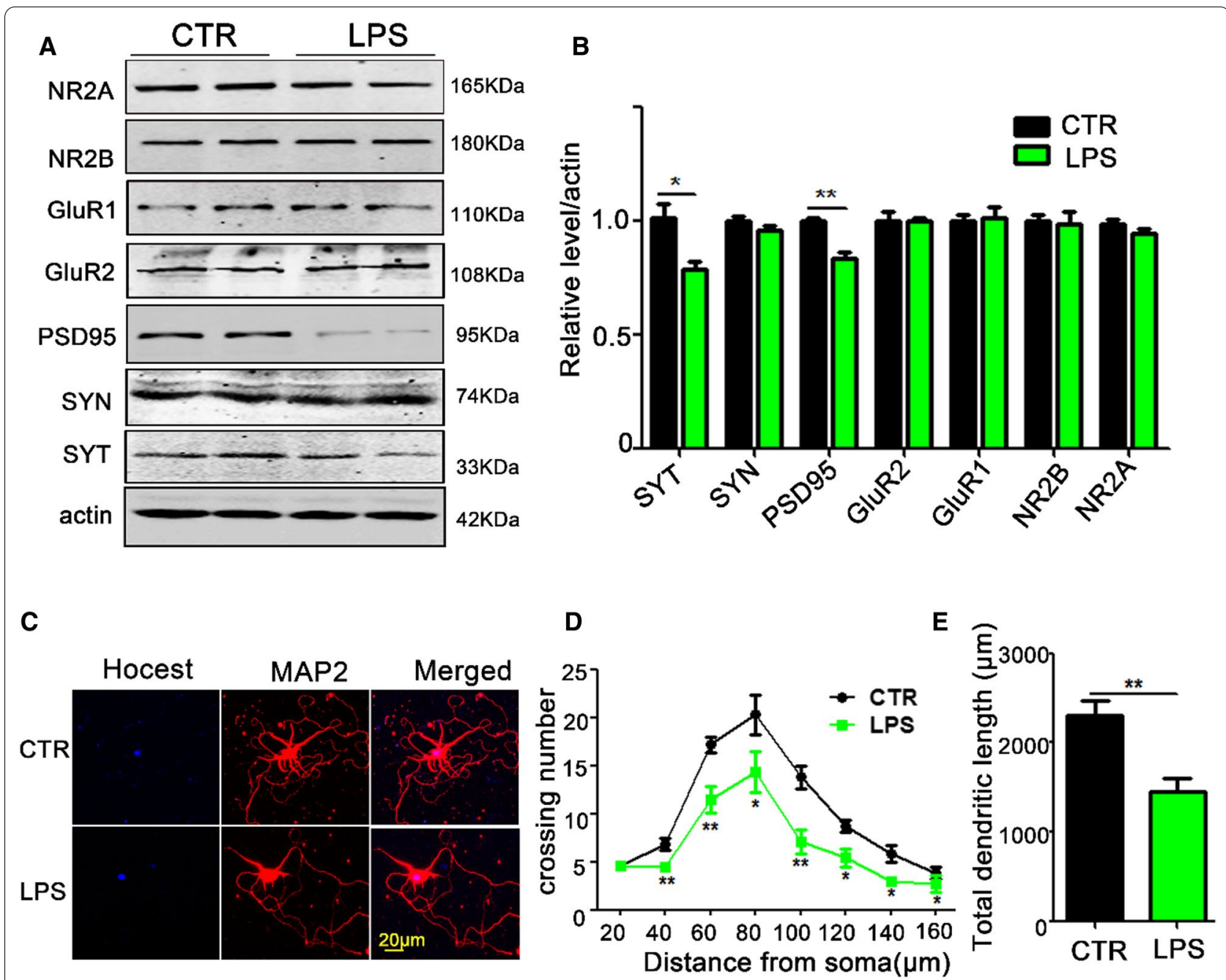

Fig. 3 LPS treatment results in synaptic dysfunction in rat primary hippocampal neurons. Western blotting (A) from homogenized primary hippocampal neurons showed the levels of synaptic protein detected by immunoblotting (B), $n=3$. C-E Rat primary hippocampal neurons (DIV7) were incubated with LPS $(1 \mu \mathrm{g} / \mathrm{ml})$ for $6 \mathrm{~h}$, and the dendritic morphology of hippocampal primary neurons following treatment with LPS was examined using anti-MAP2 antibody $(\mathbf{C})$. The dendritic arborization complexity was tested by sholl analysis (D), and the total dendritic length was measured $(\mathbf{E}), \mathrm{n}=10$. $p$ value significance is calculated from a $T$-test, and data are presented as mean $\pm \mathrm{SEM} .{ }^{*} p<0.05,{ }^{* *} p<0.01$ vs control group 


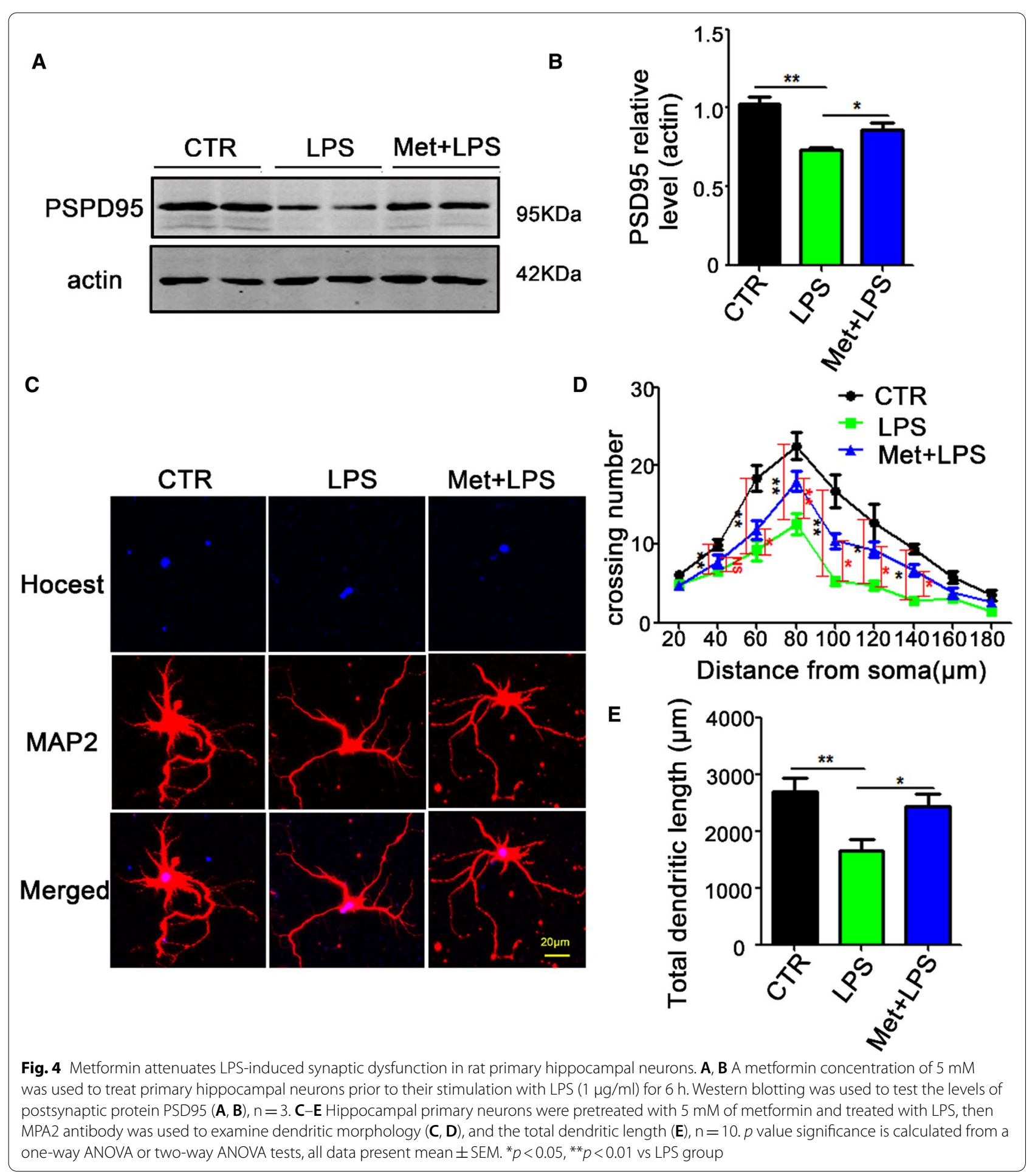

increased in metformin pretreated neurons prior to LPS when compared with the LPS group (Fig. 4D), as well as an increase in the total dendritic length (Fig. 4E). These data indicate that the metformin rescued the LPSinduced synaptic dysfunction, supporting that metformin might be a potential therapeutic agent for neuroinflammation-induced neurodegenerative diseases.

To investigate the effect of metformin on cognition and the molecular mechanisms in treated and untreated control animals. $5 \mathrm{mM}$ and $10 \mathrm{mM}$ metformin were injected 
into the lateral ventricles. We firstly performed behavior tests. NOR test results showed a significant increase in the curiosity toward new things in the metformin group when compared with the normal untreated rats (Additional file 1: Fig. S1A, B). Fear condition results showed that the freezing duration after metformin treatment was significantly higher than the untreated control group at $24 \mathrm{~h}$ (Additional file 1: Fig. S1D), but there was no significant difference at $2 \mathrm{~h}$ (Additional file 1: Fig. S1C). Finally, Morris water maze (MWM) results showed that there were no significantly difference in latency during the 5 training days (Additional file 1: Fig. S1E). On the day 6 , with the platform removed to test the spatial memory, both the time in the target quadrant (Additional file 1: Fig. S1F) and the number of target platform crossing were increased (Additional file 1: Fig. S1G) compared with the control group. Moreover, the electrophysiology experiment results showed that metformin enhanced the slope of field excitatory postsynaptic potential (fEPSP) after high-frequency stimulation (HFS) compared with the untreated control group (Additional file 1: Fig. S1H, I). Together, these data suggest that metformin might enhance cognitive function and synaptic plasticity.

\section{Metformin alleviates LPS-induced cognitive impairments and improves synaptic plasticity}

We have shown that metformin rescued the LPS-induced alteration in synaptic protein as well as restoring dendritic complexity in rat primary hippocampal neurons. Moreover, metformin enhances cognitive function and synaptic plasticity in rats. We thus, hypothesized that metformin might alleviate LPS-induced cognitive impairments. To investigate on it, 30 healthy SD rats were randomly divided into three groups. The control group was injected with normal saline intraperitoneally $(1 \mathrm{ml} /$ $\mathrm{Kg} / \mathrm{i} . \mathrm{p}, \mathrm{n}=10$ ) for $6 \mathrm{~h}$, and the model group (LPS group) was injected with LPS intraperitoneally $(830 \mu \mathrm{g} / \mathrm{Kg} / \mathrm{i} . \mathrm{p}$, $\mathrm{n}=10$ ). In the intervention group (Met + LPS group), $10 \mathrm{mM}$ metformin was injected into the lateral ventricle, and LPS was injected intraperitoneally for $6 \mathrm{~h}$ prior to behavioral tests. Behavioral, electrophysiological, and biochemical tests were performed at $6 \mathrm{~h}$ after injection (Fig. 5A). The open-field experiment results showed no significant difference in the total distance among all three groups (Fig. 5B). However, the novelty recognition experiment results showed that the Met + LPS group has a significant increase in the duration to explore new things for both $2 \mathrm{~h}$ (Fig. 5C) and 24 h (Fig. 5D). Next, fear condition experiment results showed that when compared with the LPS group, freezing duration was significantly improved in the Met + LPS group at $2 \mathrm{~h}$ (Fig. 5E) and $24 \mathrm{~h}$ (Fig. 5F). Also, Morris water maze (MWM) was used to test memory and learning abilities, and the results showed that the Met+LPS group exhibited significantly decreased latency to find the hidden platform (Fig. 5G). On the day 6 , we removed the platform to test the spatial memory, the time in the target quadrant of the Met + LPS group was increased, compared with the LPS group (Fig. 5H), as well as increase in the number of target platform crossing (Fig. 5I). These data suggest that metformin is effective in restoring learning and memory impairments in LPS rats.

We further want to explore whether metformin could improve synaptic plasticity. The LTP test showed that in LPS group, the slope of fEPSP was decreased after high frequency stimulation when compared with the control group. However, the slope of fEPSP was significantly rescued in the Met + LPS group (Fig. 5J, K).In addition, western blotting was used to detect synaptic proteins in the hippocampus (Fig. 5L), and found that the levels of SYN (Fig. 5M) and NR2B (Fig. 5N) in the Met+LPS group were both similar to control group while they were significantly reduced in the LPS group. These results suggest that metformin could reduce LPS-induced synaptic damage and improve cognitive impairments.

\section{Metformin pretreatment attenuates the release of inflammatory factors via preventing the activation of the NF-KB pathway}

LPS treatment upregulates the release of inflammatory factors via activating NF- $\mathrm{kB}$ pathway.

Neuroinflammation induces or exacerbates neurodegenerative diseases via activation of microglia cells, which releases various cytokines and other immune factors [9, 33]. To explore whether LPS can induce the release of these various immune factors, we used enzyme-linked immunosorbent assay (ELISA) Kits to detect these factors. The results showed that the LPS could cause a significant increase in the inflammatory cytokines, such as IL-1 $\beta$ (Additional file 1: Fig. S2A), IL-6 (Additional file 1: Fig. S2B), TNF- $\alpha$ (Additional file 1: Fig. S2C), which can trigger acute inflammatory reactions. Interestingly, it was shown that activation of NF- $\mathrm{kB}$ mediates the translocation of p65 from the cytoplasm to the nucleus, which in turn mediates immune responses and the release of proinflammatory mediators. Therefore, we further explored whether LPS upregulated NF- $\mathrm{kB}$ pathway. To this end, we performed western blotting and the results showed that NF-kB p65 subunit has translocated from cytoplasm (Additional file 1: Fig. S2D, E) to nucleus (Additional file 1: Fig. S2F, G) after 6 h of LPS treatment. These results suggest that LPS can activate the NF- $\mathrm{kB}$ signaling pathway and then upregulate the release of inflammatory factors.

We have proposed that LPS leads to nucleus translocation of NF-kB subunit and triggers the expression of various pro-inflammatory mediators. To explore the 


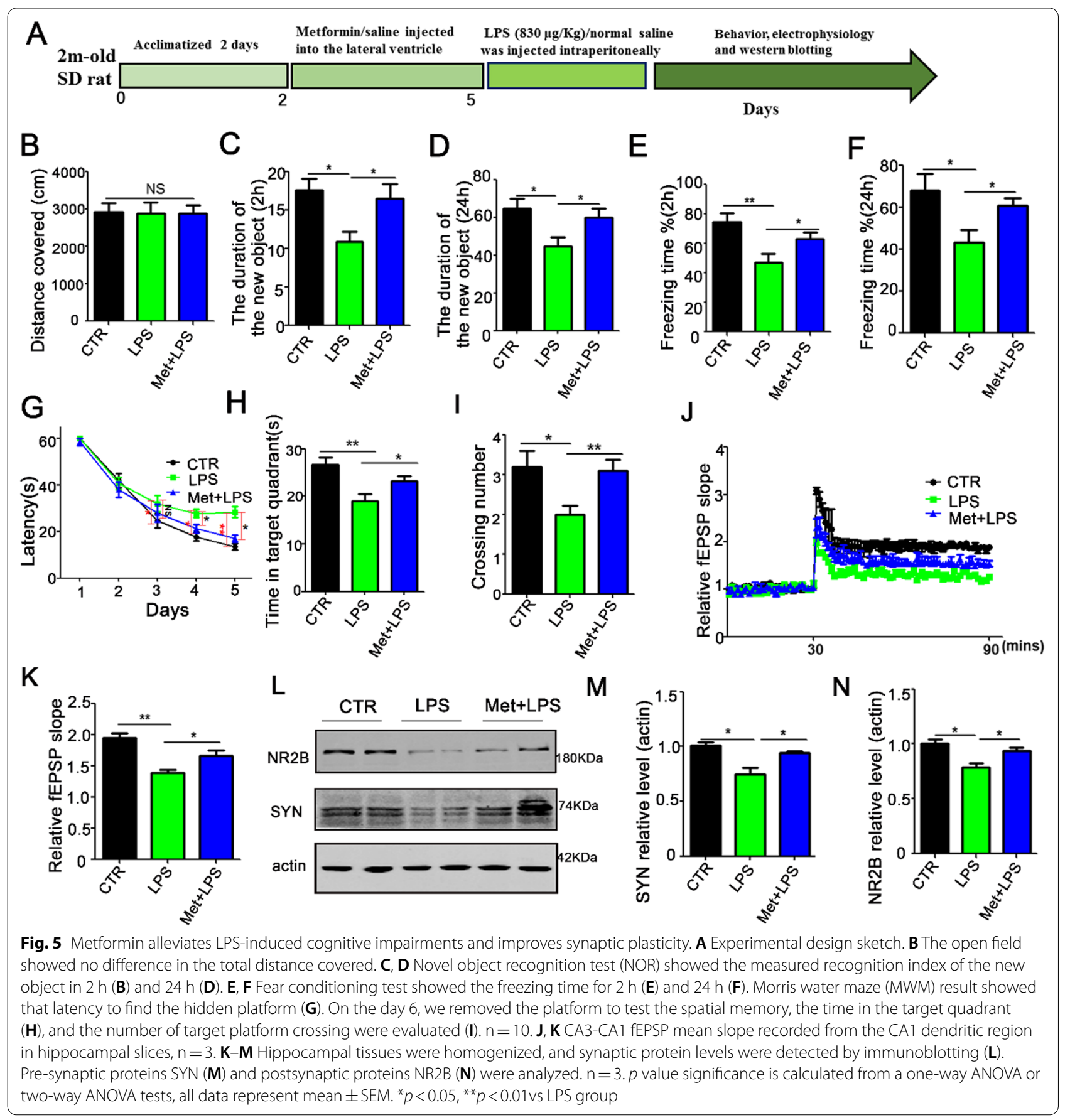

mechanism of restorative effect of metformin on learning and memory in Met + LPS rats, enzyme-linked immunosorbent assay (ELISA) Kits were used to detect inflammatory factors. The results showed that metformin can significantly inhibit the release of inflammatory factors, such as IL-1 $\beta$ (Fig. 6A), IL-6 (Fig. 6B), and TNF- $\alpha$ (Fig. 6C). We also separate cytosolic and nuclear fractions to explore the effect of metformin on the nucleus translocation of NF- $\mathrm{kB}$ by western blotting. The results showed that in the cytosolic fraction from Met + LPS group, the protein level of NF- $\mathrm{KB}$ was significantly increased compared to the LPS group (Fig. 6D, E). Furthermore, the nuclear protein level of NF- $\mathrm{kB}$ was decreased in Met + LPS group compared to the LPS group (Fig. 6F, G). These data suggest that metformin 


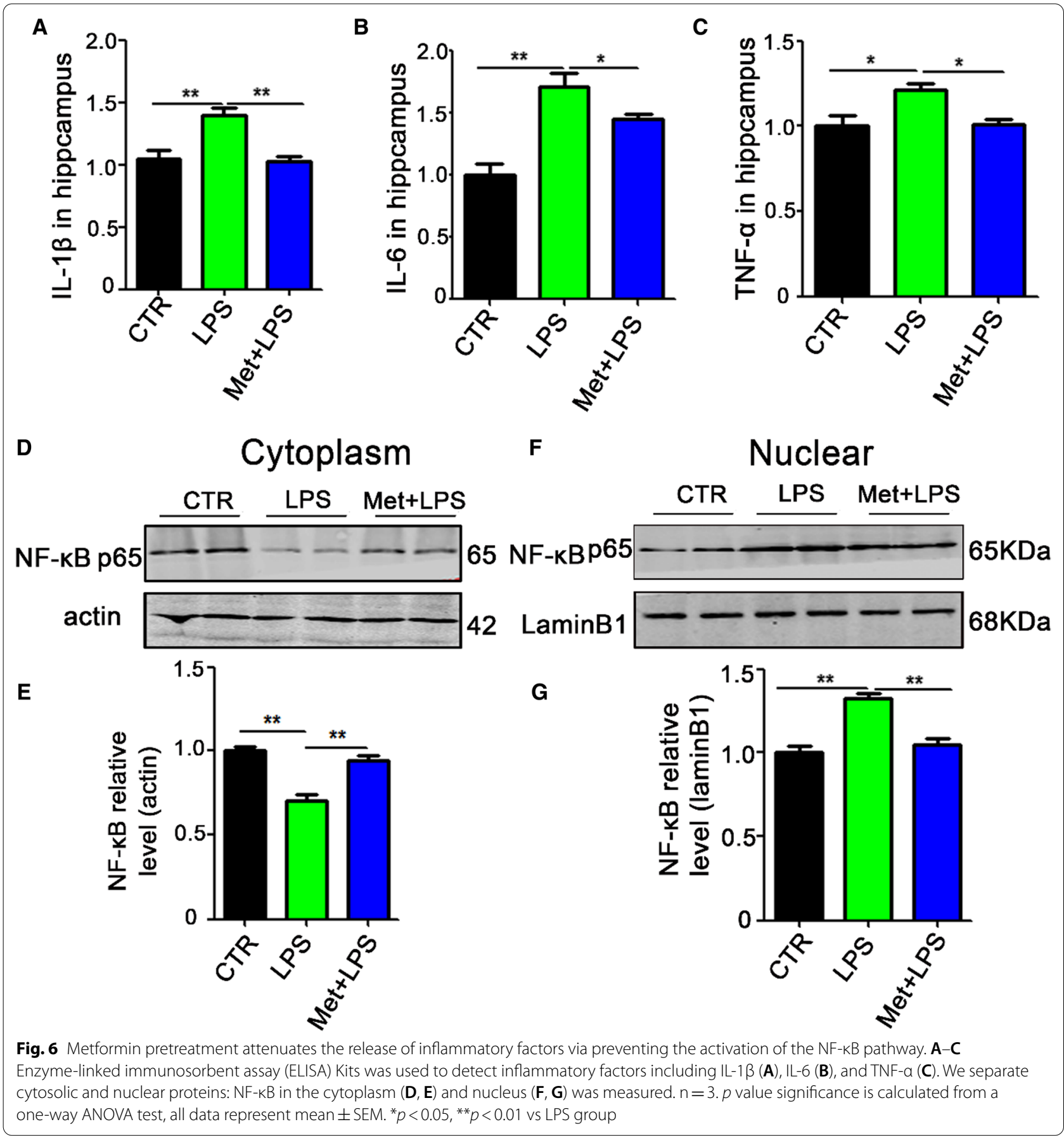

attenuates LPS-induced neuronal injury and cognitive impairments by blocking NF-kB pathway.

\section{Discussion}

Neuroinflammation is considered to be an important risk factor for the development of neurodegenerative diseases, characterized by cognitive dysfunction, neuronal damage, and apoptosis $[35,36]$. It has been reported that neuroinflammation could result in $\operatorname{cog}$ nitive impairments as a result of nuclear retention of NF- $\mathrm{kB}$ p 65 and release of inflammatory cytokines. Therefore, in order to explore NF- $\mathrm{kB}$ activation and cognitive function, LPS was used in SD rats and primary hippocampal neurons to induce inflammatory response. We found that LPS treatment triggered NF- $\mathrm{KB}$ activation. Western blotting results showed that 
LPS treatment upregulated the translocation of p65 from the cytoplasm to the nucleus.

Metformin obviously decreased LPS-induced nucleus NF- $\mathrm{kB}$ p 65 translocation. Moreover, the expression of TNF-a, IL-1 $\beta$, IL- 6 were also significantly decreased by metformin. However, it remains to be confirmed whether metformin alleviates synaptic damage and cognitive impairments. To address this, primary hippocampal neurons were incubated with or without metformin prior to LPS treatment. The results showed that LPS significantly increased neuronal injury. While the pretreatment of primary neurons with metformin obviously reduce neuronal injury. In addition, rats that were intraperitoneally injected with LPS following intraventricular injection with metformin showed enhanced cognitive function compared to those with LPS injection alone.

\section{Conclusion}

Conclusively, our data strongly support the notion that metformin attenuates LPS-induced synaptic dysfunction and cognitive impairments in rats by blocking NF- $\mathrm{KB}$ pathway. To our knowledge, this is the first study to explore the mechanism underlying the neuron damage caused by NF- $\mathrm{kB}$ pathway alterations. The present findings provide an insight that further clarify the mechanism of the neuroinflammation-induced neurotoxicity.

\begin{abstract}
Abbreviations
PD: Parkinson's disease; MS: Multiple sclerosis; AD: Alzheimer's disease; LPS: Lipopolysaccharides; SD: Sprague-Dawley; Hip: Hippocampus; Met: Metformin; NO: Nitric oxide; ROS: Reactive oxygen species; LTP: Long-term potentiation; SC: Schaffer collateral; fEPSP: Field excitatory postsynaptic potential; HFS: High-frequency stimulation; PSD95: Postsynaptic density protein 95; SYN: Synapsin; SYT: Synaptophysin; ELISA: Enzyme-linked immunosorbent assay; IL-1 $\beta$ : Interleukin-1 $\beta$; IL-6: Interleukin-6; TNF-a: Tumor necrosis factor-a; i.p: Intraperitoneally; CA1: Cornu Ammonis area 1; CA3: Cornu Ammonis area 3; MWM: Morris water maze; NOR: Novel objective recognition.
\end{abstract}

\section{Supplementary Information}

The online version contains supplementary material available at https://doi. org/10.1186/s12868-021-00678-5.

Additional file 1: Figure S1. Metformin might be beneficial to cognitive function and synaptic plasticity. Figure S2. LPS treatment upregulates the release of inflammatory factors via activating NF-KB pathway.

\section{Acknowledgements}

We thank to Dr. Xiaochuan Wang (Tongji Medical College, Huazhong University of Science and Technology) for providing experimental platform. We thank Dr. Gang Wu (Tongji Medical College, Huazhong University of Science and Technology) for the reagents procurement.

\section{Authors' contributions}

CG and CZ planned, organized and designed all experiments and results, including the writing of the manuscript. BP and WZ planned and performed all experiments, analyzed the data. ZQ performed assisted with the manuscript preparation. All authors read and approved the final manuscript.

\section{Funding}

This work was supported by Grants from General Project of Hubei Provincial Health and Family Planning Commission (MJ2017M014).

\section{Availability of data and materials}

The datasets used and/or analyzed during the present study are available from the corresponding author on reasonable request.

\section{Declarations}

\section{Ethics approval and consent to participate}

This study was approved by the Animal Care Committees of T the Ethics Committee of Renmin Hospital, Wuhan University in accordance with international regulations. The study is reported in accordance with ARRIVE guidelines.

\section{Consent for publication}

Not applicable.

\section{Competing interests}

All authors declare no conflict of interest in this current study.

\section{Author details}

'Department of Critical Care Medicine, Renmin Hospital of Wuhan University, Wuhan, China. ${ }^{2}$ Department of Neurology, Renmin Hospital of Wuhan University, Wuhan, China. ${ }^{3}$ Department of Critical Care Medicine, Tianyou Hospital Affiliated to Wuhan University of Science and Technology, Wuhan, China.

Received: 8 August 2021 Accepted: 11 November 2021

Published online: 26 November 2021

\section{References}

1. Fourrier C, Remus-Borel J, Greenhalgh AD, Guichardant M, BernoudHubac N, Lagarde M, Joffre C, Laye S. Docosahexaenoic acid-containing choline phospholipid modulates LPS-induced neuroinflammation in vivo and in microglia in vitro. J Neuroinflammation. 2017;14(1):170.

2. Kennedy PG. Viruses, apoptosis, and neuroinflammation - a doubleedged sword. J Neurovirol. 2015;21(1):1-7.

3. Qiu AW, Liu Z, Guo J, Peng YP. Relationship between neuroinflammation and neurodegenerative diseases. Sheng Li Ke Xue Jin Zhan. 2011:42(5):353-8.

4. Vivekanantham S, Shah S, Dewji R, Dewji A, Khatri C, Ologunde R. Neuroinflammation in Parkinson;s disease: role in neurodegeneration and tissue repair. Int J Neurosci. 2015;125(10):717-25.

5. Gelders G, Baekelandt V, Van der Perren A. Linking neuroinflammation and neurodegeneration in Parkinson's disease. J Immunol Res. 2018:2018:4784268

6. Nazem A, Sankowski R, Bacher M, Al-Abed Y. Rodent models of neuroinflammation for Alzheimer's disease. J Neuroinflammation. 2015;12:74.

7. Finneran DJ, Nash KR. Neuroinflammation and fractalkine signaling in Alzheimer's disease. J Neuroinflammation. 2019;16(1):30.

8. Filippi M, Bar-Or A, Piehl F, Preziosa P, Solari A, Vukusic S, Rocca MA. Multiple sclerosis. Nat Rev Dis Primers. 2018;4(1):43.

9. Bjelobaba I, Savic D, Lavrnja I. Multiple sclerosis and neuroinflammation: the overview of current and prospective therapies. Curr Pharm Des. 2017:23(5):693-730

10. Campbell JM, Stephenson MD, de Courten B, Chapman I, Bellman SM, Aromataris E. Metformin and Alzheimer's disease, dementia and cognitive impairment: a systematic review protocol. JBI Database Syst Rev Implement Rep. 2017;15(8):2055-9.

11. Ashraghi MR, Pagano G, Polychronis S, Niccolini F, Politis M. Parkinson's disease, diabetes and cognitive impairment. Recent Pat Endocr Metab Immune Drug Discov. 2016;10(1):11-21.

12. Zhang QQ, Li WS, Liu Z, Zhang HL, Ba YG, Zhang RX. Metformin therapy and cognitive dysfunction in patients with type 2 diabetes: a meta-analysis and systematic review. Medicine. 2020;99(10): e19378. 
13. Zhao J, Bi W, Xiao S, Lan X, Cheng X, Zhang J, Lu D, Wei W, Wang Y, Li H, et al. Neuroinflammation induced by lipopolysaccharide causes cognitive impairment in mice. Sci Rep. 2019;9(1):5790.

14. Lopes PC. LPS and neuroinflammation: a matter of timing. Inflammopharmacology. 2016;24(5):291-3.

15. Shu H, Wang M, Song M, Sun Y, Shen X, Zhang J, Jin X. Acute nicotine treatment alleviates LPS-induced impairment of fear memory reconsolidation through AMPK activation and CRTC1 upregulation in hippocampus. Int J Neuropsychopharmacol. 2020;23(10):687-99.

16. DiDonato JA, Mercurio F, Karin M. NF-kappaB and the link between inflammation and cancer. Immunol Rev. 2012;246(1):379-400.

17. Hoesel B, Schmid JA. The complexity of NF-kappaB signaling in inflammation and cancer. Mol Cancer. 2013;12:86.

18. Singh SS, Rai SN, Birla H, Zahra W, Rathore AS, Singh SP. NF-kappaB-mediated neuroinflammation in Parkinson's disease and potential therapeutic effect of polyphenols. Neurotox Res. 2020;37(3):491-507.

19. Li B, Xu P, Wu S, Jiang Z, Huang Z, Li Q, Chen D. Diosgenin attenuates lipopolysaccharide-induced Parkinson's disease by inhibiting the TLR NF-kappaB pathway. J Alzheimers Dis. 2018;64(3):943-55.

20. Zhao YF, Zhang Q, Xi JY, Li YH, Ma CG, Xiao BG. Multitarget intervention of Fasudil in the neuroprotection of dopaminergic neurons in MPTP-mouse model of Parkinson's disease. J Neurol Sci. 2015;353(1-2):28-37.

21. Rigillo G, Vilella A, Benatti C, Schaeffer L, Brunello N, Blom JMC, Zoli M, Tascedda F. LPS-induced histone H3 phospho(Ser10)-acetylation(Lys14) regulates neuronal and microglial neuroinflammatory response. Brain Behav Immun. 2018;74:277-90.

22. Guo C, Liu Y, Fang MS, Li Y, Li W, Mahaman YAR, Zeng K, Xia Y, Ke D, Liu $R$, et al. omega-3PUFAs improve cognitive impairments through Ser133 phosphorylation of CREB upregulating BDNF/TrkB signal in schizophrenia. Neurotherapeutics. 2020;17(3):1271-86.

23. Yin $Y$, Gao D, Wang $Y$, Wang $Z H$, Wang $X$, Ye J, Wu D, Fang L, Pi G, Yang $Y$, et al. Tau accumulation induces synaptic impairment and memory deficit by calcineurin-mediated inactivation of nuclear CaMKIV/CREB signaling. Proc Natl Acad Sci USA. 2016;113(26):E3773-3781.

24. Ren YZ, Zhang BZ, Zhao XJ, Zhang ZY. Resolvin D1 ameliorates cognitive impairment following traumatic brain injury via protecting astrocytic mitochondria. J Neurochem. 2020;154(5):530-46.

25. Dong $H$, Tang $X$, Zhang Z, Dong C. Structural insight into lipopolysaccharide transport from the Gram-negative bacterial inner membrane to the outer membrane. Biochim Biophys Acta Mol Cell Biol Lipids. 2017;1862(11):1461-7

26. Hankins JV, Madsen JA, Giles DK, Brodbelt JS, Trent MS. Amino acid addition to Vibrio cholerae LPS establishes a link between surface remodeling in gram-positive and gram-negative bacteria. Proc Natl Acad Sci USA. 2012;109(22):8722-7.

27. Yang J, Zhao Y, Shao F. Non-canonical activation of inflammatory caspases by cytosolic LPS in innate immunity. Curr Opin Immunol. 2015;32:78-83.

28. Cheng N, Liang Y, Du X, Ye RD. Serum amyloid A promotes LPS clearance and suppresses LPS-induced inflammation and tissue injury. EMBO Rep. 2018;19(10).

29. Chakraborty A, Boer JC, Selomulya C, Plebanski M, Royce SG. Insights into endotoxin-mediated lung inflammation and future treatment strategies. Expert Rev Respir Med. 2018;12(11):941-55.

30. Lisman J, Buzsaki G, Eichenbaum H, Nadel L, Ranganath C, Redish AD. Viewpoints: how the hippocampus contributes to memory, navigation and cognition. Nat Neurosci. 2017;20(11):1434-47.

31. Opitz B. Memory function and the hippocampus. Front Neurol Neurosci. 2014;34:51-9.

32. Rolls ET. The storage and recall of memories in the hippocampo-cortical system. Cell Tissue Res. 2018;373(3):577-604.

33. Guo F, Lou Y, Feng N, Li G, Xie A, Huang X, Wang Y. Exposure to lanthanum compound diminishes LPS-induced inflammation-associated gene expression: involvements of PKC and NF-kappaB signaling pathways. Biometals. 2010;23(4):669-80.

34. Yan L, Yang J, Yu M, Lu Y, Huang L, Wang J, Lu X, Jin C, Wu S, Cai Y. Lanthanum chloride induces neuron damage by activating the nuclear factor-kappa B signaling pathway in activated microglia. Metallomics. 2019;11(7):1277-87.

35. Voet S, Srinivasan S, Lamkanfi M, van Loo G. Inflammasomes in neuroinflammatory and neurodegenerative diseases. EMBO Mol Med. 2019;11(6).
36. Stonesifer C, Corey S, Ghanekar S, Diamandis Z, Acosta SA, Borlongan CV. Stem cell therapy for abrogating stroke-induced neuroinflammation and relevant secondary cell death mechanisms. Prog Neurobiol. 2017;158:94-131.

\section{Publisher's Note}

Springer Nature remains neutral with regard to jurisdictional claims in published maps and institutional affiliations.
Ready to submit your research? Choose BMC and benefit from:

- fast, convenient online submission

- thorough peer review by experienced researchers in your field

- rapid publication on acceptance

- support for research data, including large and complex data types

- gold Open Access which fosters wider collaboration and increased citations

- maximum visibility for your research: over $100 \mathrm{M}$ website views per year

At BMC, research is always in progress.

Learn more biomedcentral.com/submissions 